\title{
Silver lining in therapy of hidradenitis suppurativa
}

\author{
Michael Schultheis ${ }^{1}$, Uwe Kirschner ${ }^{2}$, Stephan Grabbe ${ }^{1}$ and Petra Staubach ${ }^{1}$ \\ ${ }^{1}$ Department of Dermatology, University Medical Center, Mainz, Germany \\ ${ }^{2}$ Dermatology Outclinic, Mainz, Germany
}

\section{Introduction}

Hidradenitis suppurativa (HS) is an inflammatory disease of the inverse skin regions that affects about $1 \%$ of the population [1]. Abscesses and fistulas, mainly formed in the armpits and groin, cause secretion, bad smelling, and severe pain [2].

However, according to current guidelines the gold standard of HS first-line-therapy beside surgery still is long-term treatment with systemic or topical antibiotics [3], which do not always stop the disease activity, lead to well-known side effects and bear the risk of resistance development [4]. A first breakthrough in HS therapy has been achieved with Adalimumab, a monoclonal anti-TNFa antibody.

Nevertheless, there is still a significant number of patients in whom Adalimumab has little effect on the disease activity [5]. Moreover, severe side effects such as Lupus like syndrome [6] and high treatment costs have to be kept in mind.

Recent clinical trials revealed that treatment with intensed pulsed light (IPL) in combination with radiofrequency (RF) (LAight ${ }^{\star}$ ) every other week reduces the number of inflammatory HS-lesions and significantly improves the quality of life (DLQI) of those affected. Patients describe a reduction of pain, an accelerated healing process as well as an improvement of the scar structure and quality of life. The treatment can be carried out on an outpatient basis and takes 15 to 60 minutes per session [7].

\section{Case}

We present a case where the combination of IPL with RF showed convincing success in a situation where the standard therapy had failed.

A 30-year-old patient with Hurley Stage II HS presented to our clinic with abscesses in the area of the axilla and the mons pubis which have been recurring for 10 years (Figure 1A and 1B). He was a non-smoker and BMI was at 24. During the 10 years of disease, local disinfection, anti-inflammatory measures as well as orals and topical antibiotics and systemic retinoids over weeks and months did not led to a satisfactory improvement of the lesions or decrease of disease burden.

In addition to the clinical picture with numerous nodules and abscesses, the patient reported profound psychological stress due to the restriction the disease had on his social-, family- and sexual life. Since therapy with antibiotics and retinoids did not lead to an improvement, we initiated mono-therapy with RF+IPL. In the beginning of the treatment IPL + RF therapy was applied every other week. After achieving almost complete remission, the treatment interval could be extended to once a month. Up to now (after 12 month of therapy), the patient is highly satisfied with a reported DLQI and pain score of both 0 and there are almost no lesions left (Figure 1C and 1D). No other treatment modality was necessary.

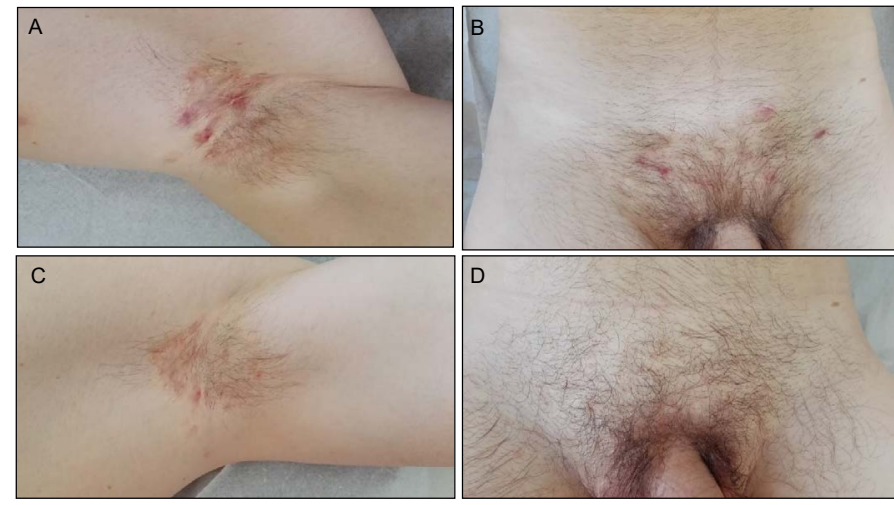

Figure 1. (A) Axilla and (B) Mons pubis - week 0; (C) Axilla and (D) Mons pubis week - 54

\section{Conclusion}

HS is chronic long lasting and often difficult to manage disease. Beside new pharmacological therapies like adalimumab and upcoming new biologicals the combination of IPL+RF is an effective long-term treatment option which is well-tolerated and can be used overall in light to moderate, but also as add-on therapy in severe cases of HS [7]. The treatment is available in Germany and some parts of Europe. A current clinical trial (EsmAiL) aiming at the implementation of highquality HS care in the outpatient setting in Germany already includes the CE-certified LAight ${ }^{\circ}$ therapy in the HS management algorithm, and upcoming new HS guidelines will need to consider this simple and effective treatment modality as well.

\section{References}

1. Jemec GB, Heidenheim M, Nielsen NH (1996) The prevalence of hidradenitis suppurativa and its potential precursor lesions. J Am Acad Dermatol 35: 191-194. [Crossref]

2. Revuz J (2009) Hidradenitis suppurativa: a comprehensive review Hidradenitis suppurativa. J Eur Acad Dermatol Venereol 23: 985-98. [Crossref]

3. Zouboulis CC, Desai N, Emtestam L, Hunger RE, Ioannides D, et al. (2015) European $\mathrm{S} 1$ guideline for the treatment of hidradenitis suppurativa/acne inversa. $J$ Eur Acad Dermatol Venereol 29: 619-644. [Crossref]

4. Bettoli V, Join-Lambert O, Nassif A (2016) Antibiotic Treatment of Hidradenitis Suppurativa. Dermatol Clin 34: 81-89. [Crossref]

5. Kimball AB, Okun MM, Williams DA, Gottlieb AB, Papp KA, et al. (2016) Two Phase 3 Trials of Adalimumab for Hidradenitis Suppurativa. $N$ Engl J Med 375: 422-434. [Crossref]

${ }^{\star}$ Correspondence to: Michael Schultheis, Department of Dermatology, University Medical Center, Mainz, Germany, E-mail: Michael.Schultheis@ unimedizin-mainz.de

Received: June 18, 2020; Accepted: June 26, 2020; Published: June 29, 2020 
6. Stein JE, Patterson-Fortin J, Bodnar BE (2018) Anti-TNF therapy-induced lupus erythematosus-like syndrome in a patient treated with adalimumab for cutaneous psoriasis. BMJ Case Rep bcr2017223473. [Crossref]
7. Wilden S, Friis M, Tuettenberg A, Grabbe S, von Stebut-Borschitz E (2017) Behandlung der Hidradenitis suppurativa (Acne inversa) mit IPL plus Radiofrequenz. Journal der Deutschen Dermatologischen Gesellschaft. 15: 85.

Copyright: $(02020$ Schultheis M. This is an open-access article distributed under the terms of the Creative Commons Attribution License, which permits unrestricted use, distribution, and reproduction in any medium, provided the original author and source are credited. 